\title{
NUMERICAL EXPLORATION OF BIFURCATION PHENOMENA ASSOCIATED WITH COMPLEX INSTABILITY
}

\author{
MERCÈ OLLÉ*
}

\begin{abstract}
The Hopf-like bifurcation associated with the transition from stability to complex instability of a family of periodic orbits in a Hamiltonian system with three (or more) degrees of freedom is investigated. Numerical techniques to compute the bifurcating objects -periodic orbits, or, more generally, 2D isolated invariant tori- are presented. The evolution and further bifurcations of the $2 \mathrm{D}$ tori are described. As a model problem, we consider two 4D symplectic mappings, and, as an application, we give some results for a galactic potential.
\end{abstract}

1. Introduction. We discuss numerical methods for the following bifurcation problem: Consider a Hamiltonian system with three or more degrees of freedom, such that there is a transition of a stable family of periodic orbits to complex instability. Complex instability was described and named so by Broucke (see [3]), and further studied numerically in celestial mechanics and galactic dynamics (e.g. see $[5,6,12,13,19])$ and also in particle accelerator problems (see [8]). It has been studied by analytical techniques in $[1,2,7,15]$.

It is well known that this transition involves a Hopf-like bifurcation of direct or inverse type. The bifurcating objects are periodic orbits or $2 \mathrm{D}$ invariant tori according to whether the rotation number, is rational or irrational. An efficient numerical method for computing the bifurcating periodic orbits is a standard continuation method. In this talk, we deal with the numerical computation of the bifurcating $2 \mathrm{D}$ invariant tori.

More specifically, the evolution and further bifurcations of such tori are investigated and two different numerical methods for their computation are presented. We show that there appear two types of bifurcations of $2 \mathrm{D}$ invariant tori resembling the bifurcation of periodic orbits: the 'perioddoubling' bifurcation and a pair of asymmetric tori. In order to understand the local universal properties of such bifurcations and to show some results, two 4D symplectic mappings representing dynamical systems with three degrees of freedom are considered.

Finally, as an application, we consider a galactic potential which gives rise to a Hamiltonian system with five equilibrium points $L_{1}, L_{2}, \ldots, L_{5}$. The $L_{4}$ point is used and, when varying some parameters, the family of periodic orbits around it undergoes a transition from stability to complex instability. The Hopf-like bifurcation is of inverse type and we show the typical behaviour of $2 \mathrm{~d}$ tori close to the transition on the stable side.

${ }^{*}$ Dept. Matemàtica Aplicada I.E.T.S.E.I.B., Universitat Politècnica de Catalunya, Diagonal 647, 08028 Barcelona, Spain. E-mail: olle@ma1.upc.es 
2. Transition stability-complex instability. Symplectic mappings. It is well known that Hamiltonian systems can be transformed into symplectic mappings by means of the Poincaré sections. In 3-degree of freedom systems, there corresponds a $4 \mathrm{D}$ section space and a family of periodic orbits is reduced to a family of fixed points.

In order to describe the transition from stability to complex instability we have used two $4 \mathrm{D}$ symplectic mappings, denoted by $T_{\mathrm{s}}$ and $T_{\mathrm{t}}$, natural generalizations of the Froeschlé map,

$$
\begin{aligned}
& T_{\mathrm{s}}\left(\begin{array}{l}
x_{1} \\
x_{2} \\
x_{3} \\
x_{4}
\end{array}\right)=\left(\begin{array}{l}
D\left[x_{1}+K_{1} \sin \left(x_{1}+x_{2}\right)+L_{1} \sin \left(x_{1}+x_{2}+x_{3}+x_{4}\right)\right] \\
x_{1}+x_{2} \\
D\left[x_{3}+K_{2} \sin \left(x_{3}+x_{4}\right)+L_{2} \sin \left(x_{1}+x_{2}+x_{3}+x_{4}\right)\right] \\
x_{3}+x_{4}
\end{array}\right) \\
& T_{\mathrm{t}}\left(\begin{array}{l}
x_{1} \\
x_{2} \\
x_{3} \\
x_{4}
\end{array}\right)=\left(\begin{array}{l}
D\left[x_{1}+K_{1} \sin \left(x_{1}+x_{2}\right)+L_{1} \tan \left(x_{1}+x_{2}+x_{3}+x_{4}\right)\right] \\
x_{1}+x_{2} \\
D\left[x_{3}+K_{2} \sin \left(x_{3}+x_{4}\right)+L_{2} \tan \left(x_{1}+x_{2}+x_{3}+x_{4}\right)\right] \\
x_{3}+x_{4}
\end{array}\right)
\end{aligned}
$$

both defined $(\bmod 2 \pi)$. The dissipation parameter $D$ is only used for computing purposes. When $D<1, D=1, D>1$ the mappings are respectively volume contracting, preserving and dilating. We study the mappings with $D=1$ around the fixed point $\mathbf{x}=0$; both have the same Jacobian $A$, but different non-linear properties as described below. We restrict the parameter space by taking $L_{1}=-L_{2} \equiv L, K_{1} \equiv K$ and $K_{2}=0$; the transition takes place when $L=-K / 4 \equiv L_{\text {crit }}$ in the interval $-8<K<0$. $L$ is a varying parameter and $K$ assumed not to depend on $L$ (see [17] for a discussion of the parameters).

Striking phase structures appear at the transition from stability to complex instability. The main result is that there is a bifurcation which turns out to be a Hopf-like bifurcation with its own particular properties: the bifurcating structures may be direct (the bifurcating objects unfold on the unstable side), or they may be inverse (the bifurcating objects unfold on the stable side) as the parameter $L$ is varied. For the mapping $T_{\mathrm{s}}$ $\left(T_{\mathrm{t}}\right)$, the bifurcation is direct (inverse), and the bifurcating objects exist for $L>L_{\text {crit }}\left(L<L_{\text {crit }}\right)$.

Let $k$ be the rotation number at the transition (that is, $k$ is defined from the eigenvalue $\lambda=1 / \lambda=e^{(i 2 \pi k)}$ of the Jacobian matrix $A$ ). When the rotation number $k=p / q$ is rational, then $q$-periodic orbits may bifurcate. In accordance to the Hopf bifurcation, some of the bifurcating families of periodic orbits inherit the stability character, but there are some that do not. In [17], a detailed description of such periodic orbits and their stability properties are given.

When the rotation number $k$ is irrational, stable (unstable) invariant curves bifurcate according to the direct (inverse) case, that is, for the mapping $T_{\mathrm{s}}\left(T_{\mathrm{t}}\right)$. These invariant curves become $2 \mathrm{D}$ tori in the phase space of the Hamiltonian system of three degrees of freedom. 
2.1. Numerical methods for the computation of invariant curves. We may expect stable invariant curves for mapping $T_{\mathrm{S}}$ and $L>$ $L_{\text {crit }}$, and unstable ones for $T_{\mathrm{t}}$ and $L<L_{\text {crit }}$.

There are several algorithms for the computation of invariant curves of maps (see, for instance, $[4,10,11,22]$ for the general case, and $[9,20]$ for symplectic maps).

We recall a first method used in [17] to compute the stable invariant curves for the mapping $T_{\mathrm{s}}$. It exploits the property that they become attracting limit cycles as soon as dissipation $(D<1)$ occurs. Starting at $D<1$, the method consists of progressively increasing $D$ while controlling the convergence of the point iterates onto the invariant curves. When $D=$ 1 , we have reached the desired invariant curve. Of course, this numerical technique finds invariant curves which are necessarily stable, and it does not work for unstable ones.

We present here a method which allows to compute both stable and unstable invariant curves. This method is based on the approximation of an invariant curve by a truncated Fourier series (the first use of this method was reported in [4] and later on revisited in [22]). Suppose we have a symplectic mapping $F: R^{4} \rightarrow R^{4}$, and let us look for an invariant curve of fixed frequency $\omega$. Such invariant curve of mapping $F$ is regarded as a function $f: S^{1} \rightarrow R^{4}, 2 \pi$ periodic such that $f(\theta+\omega)=F(f(\theta))$, where $S^{1}=\left\{(r, \theta) \in R^{2}, r=1, \theta \in[0,2 \pi)\right\}$.

Thus $f(\theta)$ has a Fourier series

$$
f(\theta)=a_{0}+\sum_{k=1}^{\infty}\left(a_{k} \cos (k \theta)+b_{k} \sin (k \theta)\right)
$$

and the numerical technique consists of computing $a_{0}, a_{k}, b_{k}$, for $k=$ $1, \ldots, N$, which give an approximation to the desired invariant curve. In Figure 1, we show some computed stable invariant curves for $T_{\mathrm{s}}$, with $K=-1$ and different values of $L$.

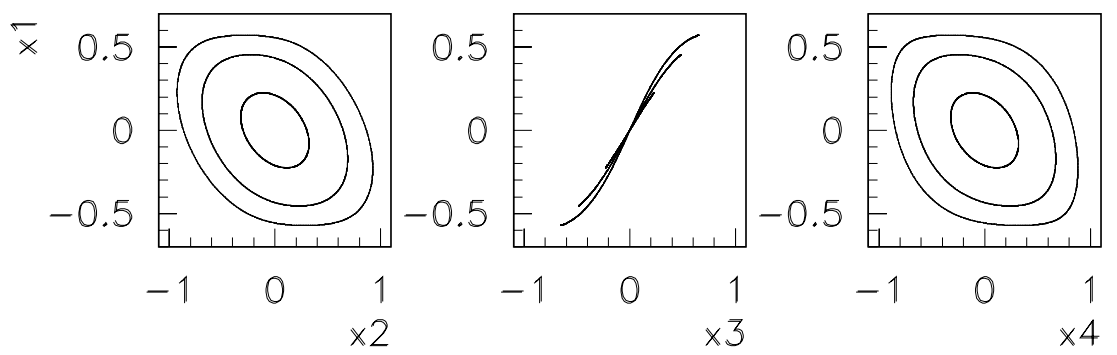

FIG. 1. Projections of the invariant curves of mapping $T_{s}, K=-1$, for values $L=0.26$ (inner one), $L=0.30, L=0.35$ (outer one). 
2.2. Other bifurcations of invariant curves. Our next goal is to follow the family of invariant curves for $T_{\mathrm{s}}$, when varying the parameter $L$ in order to find other bifurcations. When increasing the parameter $L$, for $L>L_{\text {crit }}=0.25$, the invariant curves are stable until there is a change of stability at $L=0.3965$, which gives rise to a bifurcation of "doublyperiodic" invariant curves (the invariant curve makes 2 turns before "closing' in section space).

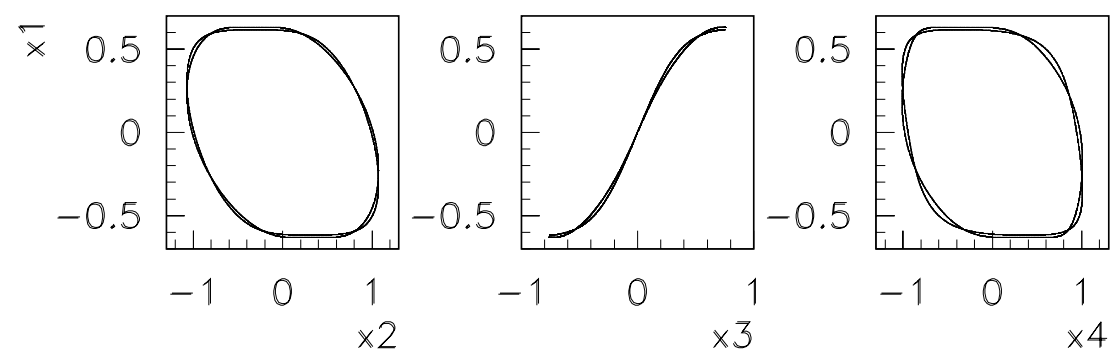

Fig. 2. 'Doubly-periodic' invariant curve of mapping $T_{s}, K=-1, L=0.395$.

Figure 2 shows a 'doubly-periodic' invariant curve. Increasing $L$, there is again a change of stability of the central family, which does not imply a new bifurcation. For $L=0.4354$ the invariant curves which were stable become again unstable and a new pitchfork bifurcation appears, however with a different behaviour: for a fixed value of $L$ two new different asymmetric invariant curves appear apart from the central one. We show in Figure 3 two asymmetric bifurcating invariant curves.

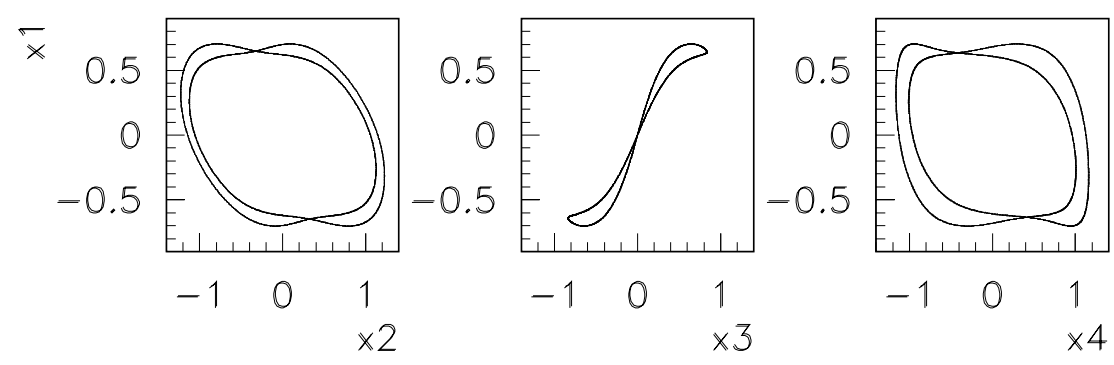

FIG. 3. Two asymmetric bifurcated invariant curves of mapping $T_{s}, K=-1$, $L=0.44$.

Final remark. So far, we have described, using mapping $T_{\mathrm{s}}$, the phenomena related to bifurcation of stable invariant $2 \mathrm{D}$ tori which unfold on the unstable side, that is, in the direct Hopf-like bifurcation. We have analyzed both the bifurcation close to the transition and further bifurcations far from it. We refer the interested reader to [14] and [17] for the consequences of the bifurcation on the phase space in a neighbourhood of the central periodic orbit close to the transition, on the unstable side. 
In a similar way, we intend to give a description of the unstable invariant curves which bifurcate on the stable side, to analyze the inverse case by means of the mapping $T_{t}$. This work is in progress.

3. Application: A Galactic potential. We consider now a Hamiltonian system, which in a frame of reference rotating about the z-axis with the frequency $\Omega$ is given by the Hamiltonian

$$
H=\frac{1}{2}\left(p_{x}^{2}+p_{y}^{2}+p_{z}^{2}\right)+\Phi(x, y, z)-\Omega\left(x p_{y}-y p_{x}\right)
$$

where $\Phi$ is a barred galaxy potential which corresponds to two mass components, a Miyamoto-Nagai disc and a triaxial $n=2$ Ferrers bar. $\Phi$ is a rather complicated function which we omit here as well as the constants and parameters involved (see [15] for details); we just note that the mass of the bar is a parameter denoted by $\mu$ from now on.

We present this example as a particular application of a Hamiltonian system with three degrees of freedom, with a family of periodic orbits which undergo a transition to complex instability, with a Hopf-like bifurcation of inverse type.

This Hamiltonian system has three equilibrium points $L_{1}, L_{2}$ and $L_{3}$ on the $\mathrm{x}$ axis and $L_{4}, L_{5}$ on the y axis. We consider in particular point $L_{4}$, and the family of 3-dimensional periodic orbits that exist close to $L_{4}$ for each value of $\mu$. Given $\mu$, we compute the initial conditions $\left(x, y, 0, p_{x}, p_{y}, p_{z}\right)$ of the periodic orbits of the family and we plot in Figure 4 the transition from stability to complex instability in a diagram $\mu-p_{z}$, when $\mu$ varies in the interval $(0.6215,0.6233)$.

Let us fix a value of $\mu$; we see from Figure 4 that there is a transition from stability to complex instability. In order to decide on the orientation of the Hopf-like bifurcation we consider the symplectic mapping given by the Poincaré section $z=0$, for a fixed value of $H$. We know from the behaviour of mappings $T_{\mathrm{s}}$ and $T_{\mathrm{t}}$ that, according to the orientation, the effect of the bifurcation on the neighbourhood of the central complex unstable periodic orbit is completely different: in the direct case it confines the chaotic orbits for some time, which may be long, or in the inverse case it allows an immediate escape (see [17] for details). Thus, we take initial conditions close to the ones of a complex unstable periodic orbit and we plot the intersections of the corresponding orbit with the Poincaré section $z=0$. It turns out that they are not confined at all and the orbit escapes. Thus, the bifurcation unfolds on the stable side, i.e., it is of inverse type.

We have not yet followed the 2D unstable tori which bifurcate on the stable side. However, it is interesting to see the effect of the bifurcation on the invariant tori surrounding the stable central family. We know from the mapping $T_{\mathrm{t}}$, that the main effect of an inverse bifurcation is to compress the stable tori inside a flattened volume. We also obtain this effect in our Hamiltonian. In order to reach the last invariant torus, we use the antidissipative procedure described in [17] for symplectic mappings and in [18] 


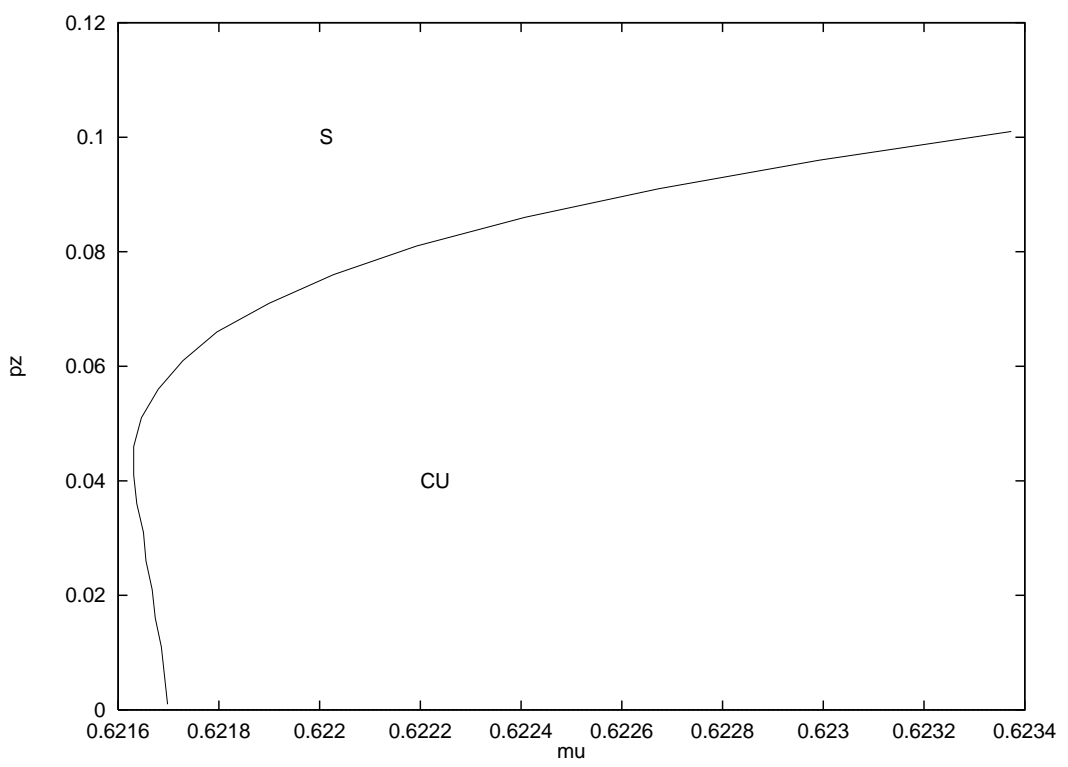

FIG. 4. Transition from stability to complex instability in a diagram $\mu-p_{z}$. The complex unstable regime is marked by $C U$, the stable one by $S$.

for galactic potentials. It consists of perturbing the equations of motion by an anti-dissipative term (i.e. dilating phase-space volumes with time) proportional to a factor $D$ slightly larger than 1 but conserving the initial Hamiltonian value $h$. We take initial conditions of an orbit belonging to the stable region; for $D=1$, the Hamiltonian case, the orbit lies on a torus (see Figure 5a). For $D=1.00001$, the consequents on the section $z=0$ explore larger and larger tori until they reach the last one. Then there is a sudden escape as visible on the last outer points in Figure 5b. An upper bound of the extent of the invariant tori is provided by the envelope of the accumulated points just before the escape.

Another particular application with a Hopf-like bifurcation but of direct type is the circular 3D Restricted three-body Problem (close to the Lagrangian point $L_{5}$ ). The intricacies of the dynamics due to this bifurcation is being studied (see [16]).

Acknowledgements. I thank Prof. D. Pfenniger for introducing me into this subject and for many useful discussions with him, Prof. A. Jorba for the suggestion of the numerical method to compute 2D tori by means of Fourier approximations, and Prof. E. J. Doedel for his comments on the bibliography. This work has been supported by CIRIT grant no. GRQ9600105. 


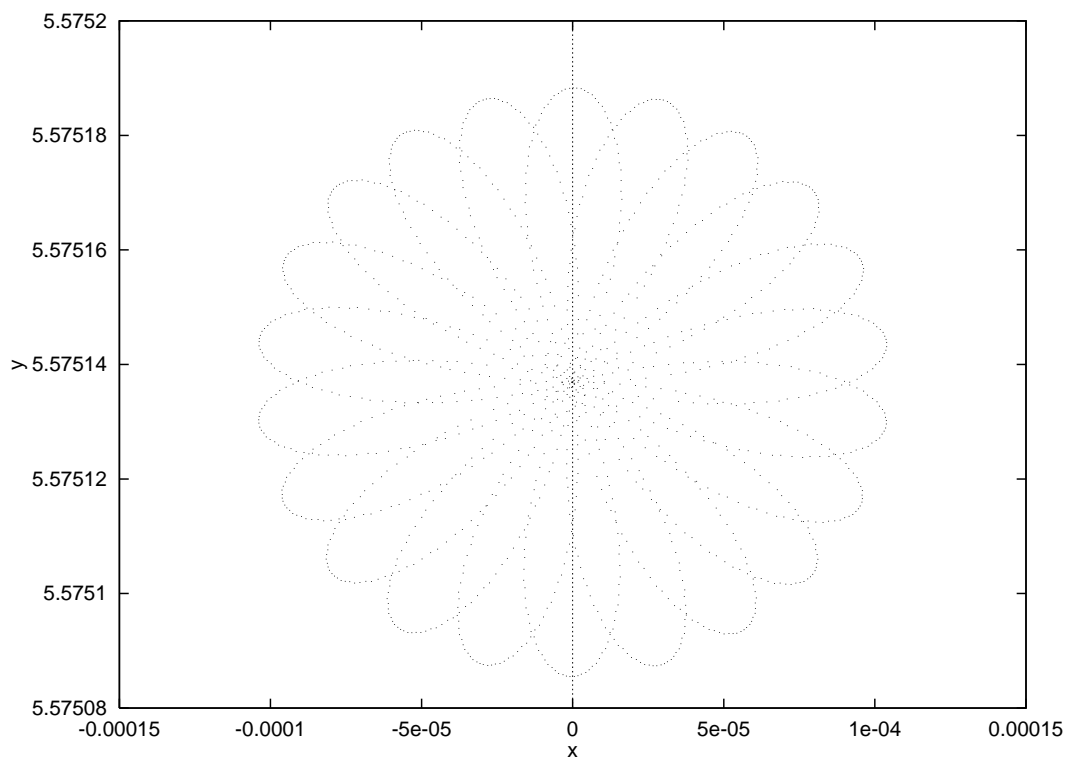

(a)

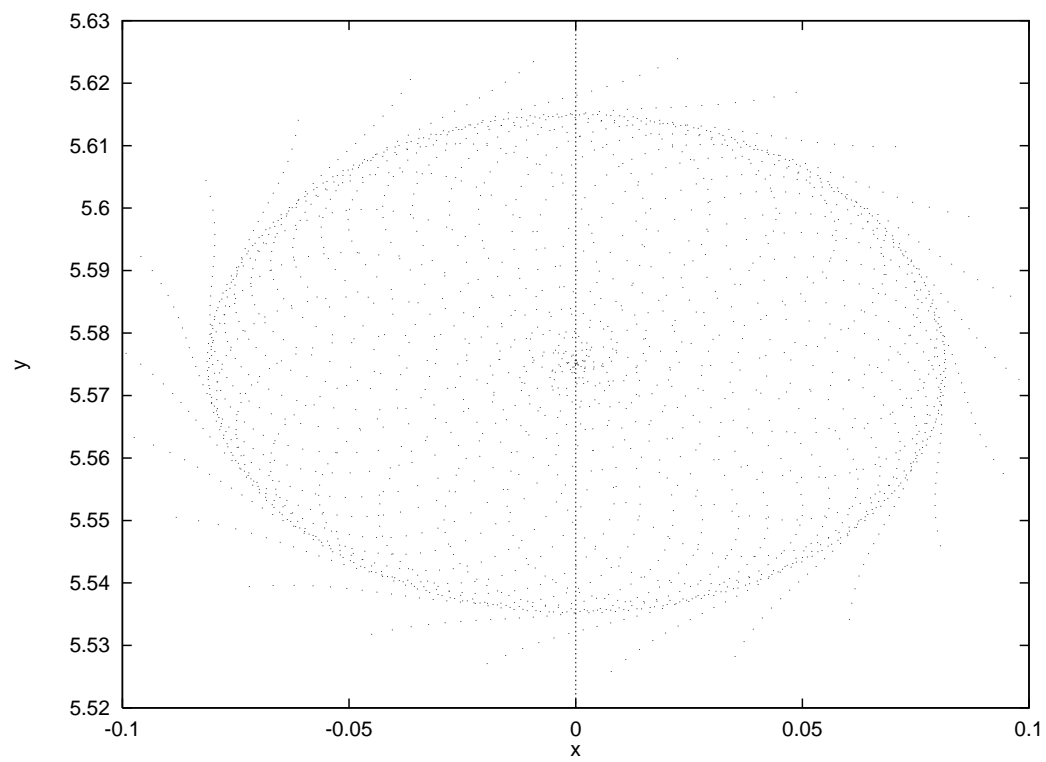

(b)

FIG. 5. Top. $(x, y)$ projections of the consequents of an orbit in the Poincaré section $z=0, p_{z}>0$ belonging to the stable region. Bottom. Envelope of the stable invariant tori around the central family obtained with the anti-dissipative method ( $D=1.00001)$. 


\section{REFERENCES}

[1] Bridges, T.J., Math. Proc. Camb. Phil. Soc., 109, 375-403 (1991).

[2] Bridges, T.J., Cushman, R.H., Mackay, R.S., Fields Institute Communications, vol. 4, 61-73 (1995)

[3] Broucke, R., AIAA J., 7, 1003-1009 (1969).

[4] Chan, Tze Ngon, Numerical Bifurcation Analysis of Simple Dynamical Systems., Ph.D Thesis, 1983.

[5] Contopoulos, G., Magnenat, P., Celest. Mech., 37, 387 (1985).

[6] Hadjidemetriou, J.D., Cel. Mech., 12, 255-276 (1975).

[7] Heggie, D.G., Cel. Mech., 35, 357-382 (1985).

[8] Howard, J.E., Lichtenberg, A.J., Lieberman, M.A., Cohen, R.H., Physica, 20D, 259-284 (1986).

[9] Jorba, A., Llave, R. Dela, Zou, M., 'Lindstedl series for lower dimensional tori', to appear in Hamiltonian systems with three or more degrees of freedom, C. Simó (Ed.), NATO adv. Sci. Inst. Ser. C phys Sci., Kluwer Acad. publ. Dordrecht, Holland, 1998.

[10] Kaas-Petersen, Chr., Physica, 25D, 288-306 (1987).

[11] Kevrekiois, I.G., Aris, R., Schmidt, L.D., Pelikan, S., Physica, 16D, 243-251 (1985).

[12] Magnenat, P., Cel. Mech., 28, 319-343 (1982).

[13] Martinet, L., Pfenniger, D., Astron. Astrophys., 173, 81-85 (1987).

[14] Ollé, M., Pfenniger, D., preprint 'Bifurcation at complex instability', to appear in Hamiltonian systems with three or more degrees of freedom, C. Simó (Ed.), NATO adv. Sci. Inst. Ser. C phys Sci., Kluwer Acad. publ. Dordrecht, Holland, 1998.

[15] Ollé, M., Pfenniger, D., preprint 'The vertical orbital structure around the Lagrangian points in barred galaxies', to appear in Astron. Astrophys, in 1998.

[16] Ollé, M., PACha, J.R., Complex instability in the RTBP, in progress.

[17] Pfenniger, D., Astron. Astrophys., 150, 97-111 (1985).

[18] Pfenniger, D., Astron. Astrophys., 150, 112-128 (1985).

[19] Pfenniger, D., Astron. Astrophys., 180, 79-93 (1987).

[20] Simó, C., 'Effective Computations in Hamiltonian Dynamics', Cent ans après les Méthodes Novvelles de H. Poincaré Société Mathématique de France, 1-23 (1996).

[21] Van Der Meer, The Hamiltonian Hopf Bifurcation. Lecture Notes in Math., 1160, Springer-V (1985).

[22] Veldhvuizen, M. van, On invariant curves and their numerical approximation. Computational ordinary differential equations, S.O. Fatula, ed., University Press. PLC Ibadan, 251-263 (1992). 